\title{
Improved 12-Lead ECG Reconstruction from Lead Sub Sets by Dynamic Selection of Frontal Leads
}

\author{
SP Nelwan ${ }^{1}$, DD Finlay ${ }^{2}$, TB van Dam $^{1}$, SH Meij ${ }^{1}$ \\ ${ }^{1}$ Erasmus MC, Rotterdam, The Netherlands \\ ${ }^{2}$ University of Ulster, Belfast, UK
}

\begin{abstract}
Patient monitoring with 12-lead ECG subsets typically uses the independent frontal leads I and II and any number of the six precordial leads to reconstruct the unrecorded ECG leads. However, variations of QRS amplitudes in leads I or II may have an effect on the signal to noise ratio of the reconstructed leads. The aim of this study was to develop and evaluate a dynamic frontal lead selection method (DFLS) to improve ECG reconstruction. We compared the DFLS method for general (GEN) and patient-specific (PS) reconstruction with a lead subset I, II, $V_{2}$, and $V_{5}$. For GEN reconstruction, a data set of 2372 diagnostic 12-lead ECGs obtained from subjects with chest pain suggestive of acute myocardial infarction was used. For PS, a data set of 71 continuous 12-lead PCI recordings was used. Reconstruction accuracy was assessed with correlation coefficients and root mean square errors. This study showed that the DFLS method increases GEN reconstruction performance in a subgroup with low $Q R S$ voltages. PS reconstruction shows a moderate overall performance increase.
\end{abstract}

\section{Introduction}

In recent years, patient monitoring with lead subsets of the 12-lead electrocardiogram (ECG) and subsequent reconstruction of the unrecorded leads has been studied by various research groups $[1,2,3,4,5,6,7]$. Typical reduced lead set implementations in clinical practice use the independent frontal leads I and II and two of the six precordial leads to reconstruct the four unrecorded ECG leads using general and patient-specific coefficients. For example, Drew et al. [4] proposed a lead subset with leads I, II, $V_{1}$ and $V_{5}$. With the coefficients published by Xue et al. [7], the unrecorded lead $\mathrm{V}_{2}$ can be reconstructed with $\mathrm{dV}_{2}=0.887330 * \mathrm{I}+-0.091160 * \mathrm{II}+1.578620 * \mathrm{~V}_{1}+$ $0.230214 * \mathrm{~V}_{5}$. The other unrecorded leads $\mathrm{V}_{3}, \mathrm{~V}_{4}$ and $\mathrm{V}_{6}$ are reconstructed in a similar fashion, but with different coefficients.

One of the most important challenges with the practical use of reduced lead sets in clinical settings is to minimize reconstruction errors as a result of noise propagation from a recorded lead. Noise propagation can be caused by poor lead subset selection and the computation of reconstruction coefficients.

Several studies $[1,3,4,5]$ have focused on the optimal selection of precordial leads. The choice of the frontal leads is often fixed to the limb leads I and II, as it is well-known that the remaining leads III, aVR, aVL and aVF can be mathematically derived from I and II. Based on the ECG lead theory and the Einthoven triangle, any combination of two frontal leads can be used to generate the remaining leads. For example, instead of I and II, Wang et al. [6] used frontal leads II and III and Schreck et al. [2] proposed perpendicular frontal leads I and aVF.

Depending on the average direction of the ventricular activation in the frontal plane, QRS amplitudes in leads I or II may be diminished and may have an effect on the signal to noise ratio of the reconstructed leads. For example, Figure 1 shows a frontal QRS axis of $94^{\circ}$ with a small biphasic QRS complex in lead I.

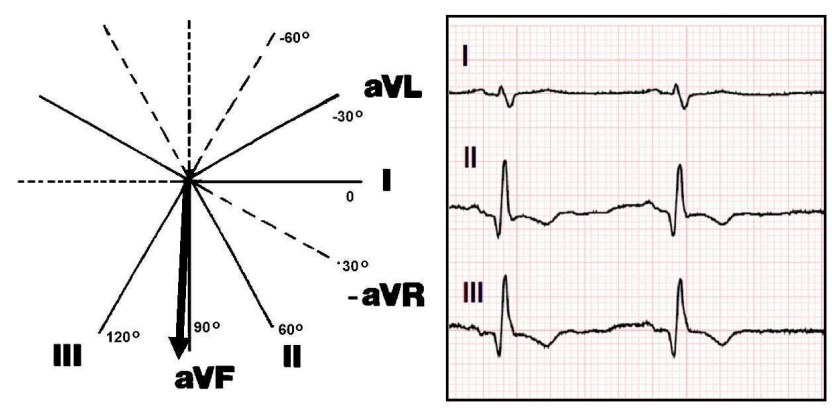

Figure 1. Frontal QRS axis of $94^{\circ}$ and its effect on the frontal leads.

For patient-specific reconstruction, ECG leads with small amplitudes may have an effect on the computation of the reconstruction coefficients. Reconstruction coefficients for those leads will often be high to compensate for the small amplitudes. As the signal to noise ratio is low, noise in 
these recorded leads during long-term patient monitoring may propagate to the reconstructed leads.

For general reconstruction, the coefficients have been computed from a larger patient group and the effects of low amplitude leads have been averaged out. It is, however, unknown if dynamic selection of frontal leads and appropriate matrices may improve general reconstruction.

In this study, we propose and evaluate a reconstruction method by selecting a different combination of two frontal plane leads with the aim to improve ECG reconstruction.

\section{Methods}

\subsection{Data sets}

For the evaluation of general reconstruction, the data set of the REPAIR study [8] was used. The REPAIR data set contains 2372 ECGs and has been used for the development and evaluation of a computer-assisted decision algorithm for early initiation of thrombolytic therapy in the pre-hospital setting in the municipality of Rotterdam. Between 1992 and 1994, patients with chest pain suggestive of myocardial infarction were evaluated by their general practitioner, who then asked for ambulance assistance and hospital admission. After arrival of the ambulance, a 12-lead ECG was recorded by the ambulance personnel and interpreted by a computerized analysis system.

For the evaluation of patient-specific reconstruction, a second data set of 71 patients undergoing a percutaneous coronary intervention procedure $[9,10]$ was used. For each patient, two 12-lead ECGs were available. At the start of the procedure, a 5-minute 12-lead pre-PCI ECG was recorded prior to the procedure using a Siemens/Draeger patient monitor (SC7000, Andover, MA, USA). Another ECG (inflation) was marked at balloon inflation.

\subsection{Data analysis}

The ECGs were analyzed by the Modular ECG Analysis System (MEANS) [11]. MEANS computes averaged beats for each lead and provides global measurements, including the heart rate and frontal QRS axis, and measurements for each lead, including the $\mathrm{Q}, \mathrm{R}, \mathrm{S}$ and T peaks. A threshold of $100 \mu \mathrm{V}$ for the $\mathrm{R}$ wave and a $\mathrm{R} / \mathrm{S}$ ratio $\geq 0.4$ was used to determine if a certain lead had a low $\mathrm{QRS}$ voltage. For the frontal QRS axis, the normal range was defined as $-30^{\circ}$ to $90^{\circ}$, a leftward frontal $\mathrm{QRS}$ axis $\leq 15^{\circ}$ and a rightward QRS axis $\geq 75^{\circ}$.

The recordings were divided into a separate learning set $(n=1188)$ and test set $(n=1160)$. A total number of 24 ECGs were excluded because of electrode interchanges or extensive noise in one or more leads.

\subsection{ECG reconstruction}

General reconstruction coefficients for all combinations of at least two of the six frontal leads and precordial leads $\mathrm{V}_{2}$ and $\mathrm{V}_{5}$ were computed using least-squares, multiple linear regression from the REPAIR learning set. To assess reconstruction performance, the coefficients were applied to the ECGs of the test set.

Patient-specific coefficients were computed for each patient of the pre-PCI ECG data and were applied to the inflation ECG of the same recording to assess patient-specific reconstruction performance.

\subsection{Dynamic frontal lead selection method}

Figure 2 shows a flow diagram of the dynamic frontal lead selection method (DFLS) for both general and patientspecific reconstruction. For each of the 15 combinations of at least two frontal leads and precordial leads $\mathrm{V}_{2}$ and $\mathrm{V}_{5}$, reconstruction coefficients are calculated from the learning set and are stored in a matrix library.

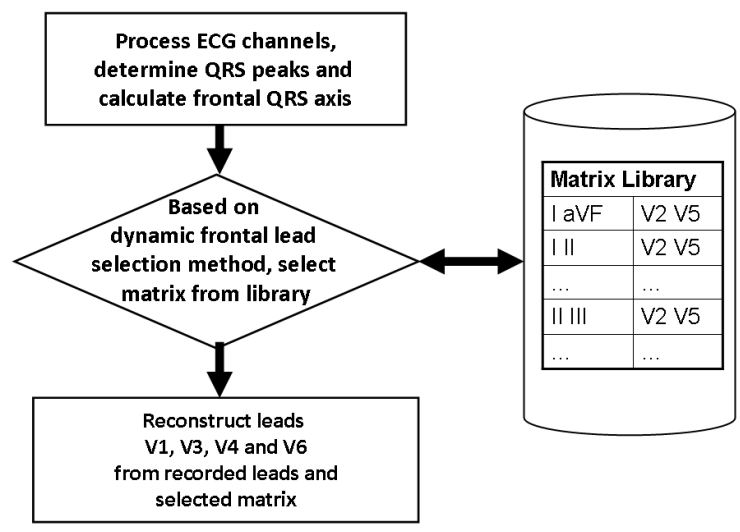

Figure 2. Schematic of the different steps for selecting the best reconstruction matrix from a library containing all combinations of at least two frontal leads and precordial leads $\mathrm{V}_{2}$ and $\mathrm{V}_{5}$.

Based on the frontal QRS axis and the classification of a low QRS in lead I or lead II, a different combination of two frontal leads is selected from the matrix library and used instead of the default lead subset I, II, $V_{2}$ and $V_{5}$.

\subsection{Performance evaluation}

Reconstruction accuracy of the dynamic frontal lead method with general and patient-specific coefficients was evaluated on the test set. To quantify the degree of similarity between the original and reconstructed lead, we computed Pearson's correlation coefficient (CC) and the root mean 
square error (RMSE). For each ECG, the average CC and RMSE of the reconstructed leads were taken and the median of the averaged CC and RMSE was used to determine the best method.

Results are presented as median (interquartile range) or average $\pm \mathrm{SD}$, where appropriate. $\mathrm{CC}$ is presented in a range from -100 to 100 , where 100 yields a perfect correlation and -100 a perfect inverse correlation. Differences in baseline characteristics were evaluated with $t$-tests and $\chi^{2}$-tests. Wilcoxon's signed rank test was used to evaluate differences between reconstruction methods. A $p$-value $<0.05$ was considered statistically significant.

\section{Results}

\subsection{General reconstruction}

The baseline characteristics of the learning and test set for the general reconstruction evaluation are presented in Table 1 . The learning and test set are similar in composition. A combined total of 28 ECGs in the test set showed low QRS voltages $(\leq 100 \mu \mathrm{V})$ in leads I or II, or both.

Table 1. Baseline characteristics of the REPAIR learning and test set.

\begin{tabular}{lcc}
\hline & $\begin{array}{c}\text { Learning Set } \\
(\mathrm{n}=1188)\end{array}$ & $\begin{array}{c}\text { Test Set } \\
(\mathrm{n}=1160)\end{array}$ \\
\hline HR (bpm) & $81 \pm 24$ & $83 \pm 24$ \\
QRS duration (ms) & $106 \pm 31$ & $108 \pm 26$ \\
Frontal QRS axis $\left(^{\circ}\right)$ & $53(10-75)$ & $50(14-76)$ \\
QRS axis $\leq 15^{\circ}(\mathrm{n}, \%)$ & $329(27.7 \%)$ & $298(25.7 \%)$ \\
QRS axis $\geq 75^{\circ}(\mathrm{n}, \%)$ & $307(25.8 \%)$ & $301(25.9 \%)$ \\
Low QRS voltage * & $18(1.5 \%)$ & $14(1.2 \%)$ \\
$\quad$ lead I $(\mathrm{n}, \%)$ & $12(1.0 \%)$ & $18(1.6 \%)$ \\
lead II $(\mathrm{n}, \%)$ &
\end{tabular}

Table 2 shows the reconstruction performance on the test set and the group of 28 recordings (LOWQRS) using lead subset I, II, $\mathrm{V}_{2}$, and $\mathrm{V}_{5}$ and the dynamic reconstruction method with the same two precordial leads. For the test set, median CC and RMSE were the same for both methods. For LOWQRS, median RMSE was lower for the DFLS method, but this was not statistically significant. The median absolute RMSE difference in LOWQRS between the standard and DFLS method was $2(0-4) \mu \mathrm{V}$ and was not statistically significant.

We also compared the performance of the lead subsets, I, aVF, $\mathrm{V}_{2}$, and $\mathrm{V}_{5}$ (Schreck [2]) and II, III, $\mathrm{V}_{2}$, and $\mathrm{V}_{5}$ (Wang [6]) on the test set. The median and interquartile range CC and RMSE of these lead subsets were identical
Table 2. Median (interquartile range) $\mathrm{CC}$ and RMSE of lead subset I, II, $\mathrm{V}_{2}$, and $\mathrm{V}_{5}$ and DFLS method using general reconstruction coefficients.

\begin{tabular}{lll}
\hline Method & $\begin{array}{l}\text { Test Set } \\
(\mathrm{n}=1160)\end{array}$ & $\begin{array}{l}\text { LOWQRS } \\
(\mathrm{n}=28)\end{array}$ \\
\hline I, II, $\mathrm{V}_{2}, \mathrm{~V}_{5}$ & & \\
CC & $95.9(92.2-97.8)$ & $93.7(86.7-95.2)$ \\
$\quad$ RMSE $(\mu \mathrm{V})$ & $91(67-125)$ & $118(74-180)$ \\
DFLS, $\mathrm{V}_{2}, \mathrm{~V}_{5}$ & & \\
CC & $95.9(92.4-97.8)$ & $93.7(87.1-95.3)$ \\
RMSE $(\mu \mathrm{V})$ & $91(66-125)$ & $115(74-173)$ \\
\hline
\end{tabular}

to the $\mathrm{CC}$ and RMSE of the lead subset I, II, $\mathrm{V}_{2}$ and $\mathrm{V}_{5}$.

\subsection{Patient-specific reconstruction}

The baseline characteristics of the pre-PCI and inflation ECGs are presented in Table 3. Table 4 shows the reconstruction performance results of the lead subset I, II, $\mathrm{V}_{2}$, and $\mathrm{V}_{5}$ and the dynamic frontal lead selection method. The median and interquartile range of CC increased only moderately. The median absolute RMSE difference between standard and DFLS was $4(0-6) \mu \mathrm{V}$ and was not statistically significant.

Table 3. Baseline characteristics of the pre-PCI and balloon inflation ECGs of the PCI data set $(n=71)$.

\begin{tabular}{lcc}
\hline & $\begin{array}{c}\text { Pre-PCI } \\
\text { ECG }\end{array}$ & $\begin{array}{c}\text { Balloon } \\
\text { Inflation ECG }\end{array}$ \\
\hline HR (bpm) & $64 \pm 11$ & $66 \pm 13$ \\
QRS duration (ms) & $105 \pm 15$ & $110 \pm 18$ \\
Frontal QRS axis ( $\left.{ }^{\circ}\right)$ & $53(-2-82)$ & $64(-4-84)$ \\
QRS axis $\leq 15^{\circ}(\mathrm{n}, \%)$ & $20(28.1 \%)$ & $19(26.7 \%)$ \\
QRS axis $\geq 75^{\circ}(\mathrm{n}, \%)$ & $27(38.6 \%)$ & $26(36.6 \%)$ \\
Low QRS voltage * & & \\
lead I (n, \%) & $4(5.6 \%)$ & $5(7.0 \%)$ \\
lead II (n, \%) & $1(1.4 \%)$ & $1(1.4 \%)$ \\
\hline
\end{tabular}

* low QRS voltage defined as $(\leq 100 \mu \mathrm{V}), \mathrm{R} / \mathrm{S}$ ratio $\geq 0.4$

Table 4. Median (interquartile range) $\mathrm{CC}$ and RMSE of lead subset I, II, $\mathrm{V}_{2}$, and $\mathrm{V}_{5}$ and the DFLS method for patient specific reconstruction.

\begin{tabular}{lll}
\hline \multirow{2}{*}{ Method } & \multicolumn{2}{c}{ Balloon Inflation $(\mathrm{n}=71)$} \\
\cline { 2 - 3 } & $\mathrm{CC}$ & $\mathrm{RMSE}(\mu \mathrm{V})$ \\
\hline I, II, $\mathrm{V}_{2}, \mathrm{~V}_{5}$ & $98.5(96.9-99.1)$ & $47(30-75)$ \\
DFLS $, \mathrm{V}_{2}, \mathrm{~V}_{5}$ & $98.7(97.2-99.1)$ & $44(30-68)$ \\
\hline
\end{tabular}




\section{Discussion and conclusions}

The presented dynamic frontal lead selection method increases reconstruction performance and signal to noise ratio in the reconstructed leads. However, the performance increase is very low, because a different combination of two frontal leads does not contain more information than is available in the standard combination of lead I and II.

For general reconstruction, the DFLS method may not increase median CC or lower median RMSE on the complete test set, but in a subgroup of ECGs with low QRS voltages, the DFLS lowers median RMSE.

For patient-specific reconstruction, higher $\mathrm{CC}$ and lower median RMSE were present in the balloon inflation set after applying the DFLS method. A possible explanation is that the DFLS method avoids overlearning of the patient's pre-PCI ECG.

Although the performance increase may be modest, the DFLS method might be appropriate for individual patients. In this study, the DFLS method increases reconstruction performance in the data set with low QRS voltages. This small group is important for the clinical acceptance of reduced lead sets. As the value of a (new) technology is often judged by a single case, any additional reduction of reconstruction errors or noise propagation may help.

The DFLS method extends the general and patientspecific reconstruction methods, but requires additional memory to store the coefficient matrices for each of the different frontal lead combinations. Also, extra processing power is required to periodically evaluate the dynamic selection of frontal leads during patient monitoring. Further improvements to the DFLS method are to investigate the use additional parameters, such as the frontal $\mathrm{T}$ axis.

\section{References}

[1] Nicklas JM, Scherer JA. US Patent No. 5,058,598: Method and Apparatus for synthesizing leads of an electrocardiogram. US Patent Office Oct 1991;600/512.

[2] Schreck DM, Tricarico VJ, Frank JD, Thielen LE, Chhibber P, Brotea C, Leber IB. Statistical methodology: VI. Mathematical modeling of the electrocardiogram using factor analysis. Acad Emerg Med Sep 1998;5(9):929-934.

[3] Nelwan SP, Kors JA, Meij SH. Minimal lead sets for reconstruction of 12-lead electrocardiograms. J Electrocardiol 2000;33 Suppl:163-166.
[4] Drew BJ, Pelter MM, Brodnick DE, Yadav AV, Dempel D, Adams MG. Comparison of a new reduced lead set electrocardiogram with the standard electrocardiogram for diagnosing cardiac arrhythmias and myocardial ischemia. J Electrocardiol 2002;35 (Suppl):13-21.

[5] Wei D, Kojima T, Nakayama T, Sakai Y. US Patent No. 6,721,591: Method of deriving standard 12-lead electrocardiogram and electrocardiogram monitoring apparatus. US Patent Office April 2004;600/512.

[6] Wang JY, Warren JW, Horacek BM. Optimal placement of dual chest leads for deriving 12-lead/18-lead electrocardiograms and vectorcardiograms. In Computers In Cardiology. IEEE, 2005; 199-202.

[7] Xue JQ. Adapting ECG morphology changes from a reduced lead set by specifically trained algorithms for acute ischemia detection. In Computers In Cardiology. IEEE, 2007; 709-712.

[8] Boersma E, Maas AC, Hartman JAM, Ilmer B, Vos J, Simoons ML. Twelve year triage and thrombolysis treatment prior to hospitalization for myocardial infarction patients in the Rotterdam area of the Netherlands: outstanding short-term and long-term results. Ned Tijdschr Geneeskd 2001;145:2029-2035.

[9] Nelwan SP, Crater SW, Green CL, Johanson P, van Dam TB, Meij SH, Simoons ML, Krucoff MW. Assessment of derived 12-lead electrocardiograms using general and patientspecific reconstruction strategies at rest and during transient myocardial ischemia. Am J Cardiol Dec 2004;94(12):15291533.

[10] Nelwan SP, Kors JA, Crater SW, Meij S, van Dam TB, Simoons ML, Krucoff MW. Simultaneous comparison of 3 derived 12-lead electrocardiograms with standard electrocardiogram at rest and during percutaneous coronary occlusion. J Electrocardiol 2008;41:230-237.

[11] van Bemmel JH, Kors JA, van Herpen G. Methodology of the modular ECG analysis system MEANS. Methods Inf Med Sep 1990;29(4):346-353.

Address for correspondence:

Stefan Nelwan

Thoraxcenter

Erasmus MC

's-Gravendijkwal 230

3015 CE Rotterdam

The Netherlands

s.nelwan@erasmusmc.nl 